\title{
Shear waves in a fluid saturated elastic plate
}

\author{
A PRADHAN, S K SAMAL and N C MAHANTI \\ Department of Applied Mathematics, Birla Institute of Technology, Mesra, \\ Ranchi 835 215, India \\ e-mail: anjana_pghorai@yahoo.co.in
}

MS received 25 February 2002

\begin{abstract}
In the present context, we consider the propagation of shear waves in the transverse isotropic fluid saturated porous plate. The frequency spectrum for SH-modes in the plate has been studied. It is observed that the frequency of the propagation is damped due to the two-phase character of the porous medium. The dimensionless phase velocities of the shear waves have also been calculated and presented graphically. It is interesting to note that the frequency and phase velocity of shear waves in porous media differ significantly in comparison to that in isotropic elastic media.
\end{abstract}

Keywords. Shear waves; porous medium; phase velocity; isotropic elastic medium.

\section{Introduction}

The dynamic behaviour of structured porous media is of great concern in many fields, such as seismology, earthquake engineering and fluid dynamics. Porous media are composite and multiphase. Their composite nature is due to the fact that the solid fraction is formed of grains whose chemical or crystalline features are often different and they are also multiphase because the solid fraction is always associated with a gas or liquid phase that occupies the voids between the grains. The microscopic heterogeneity of the porous medium induces a complex macroscopic physical behaviour sensitive to slight variations in fluid content or of the solid structure.

The propagation of Love waves in a fluid-saturated porous anisotropic layer have been discussed by Konczak (1989). Deresiewicz (1961) was the first who, using the theory of Biot (1956) for the wave propagation in a statistically isotropic fluid-saturated porous medium, studied the propagation of Love waves in a porous layer resting on an elastic, homogeneous and isotropic semi-infinite space. Chattopadhyay \& De (1983) investigated the propagation of Love waves in an isotropic, fluid-saturated porous layer with irregular interface between the layer and the lower homogeneous and isotropic half-space. Chattopadhyay et al (1986) studied the propagation of Love waves in a homogeneous, isotropic porous layer overlying an inhomogeneous half-space generated by a point source at the interface of the layer and halfspace. Chattopadhyay \& Bandopadhyay (1986) studied the shear waves in infinite monoclinic crystal plate and discussed the ferquency spectrum and the phase velocity. Chattopadhyay 


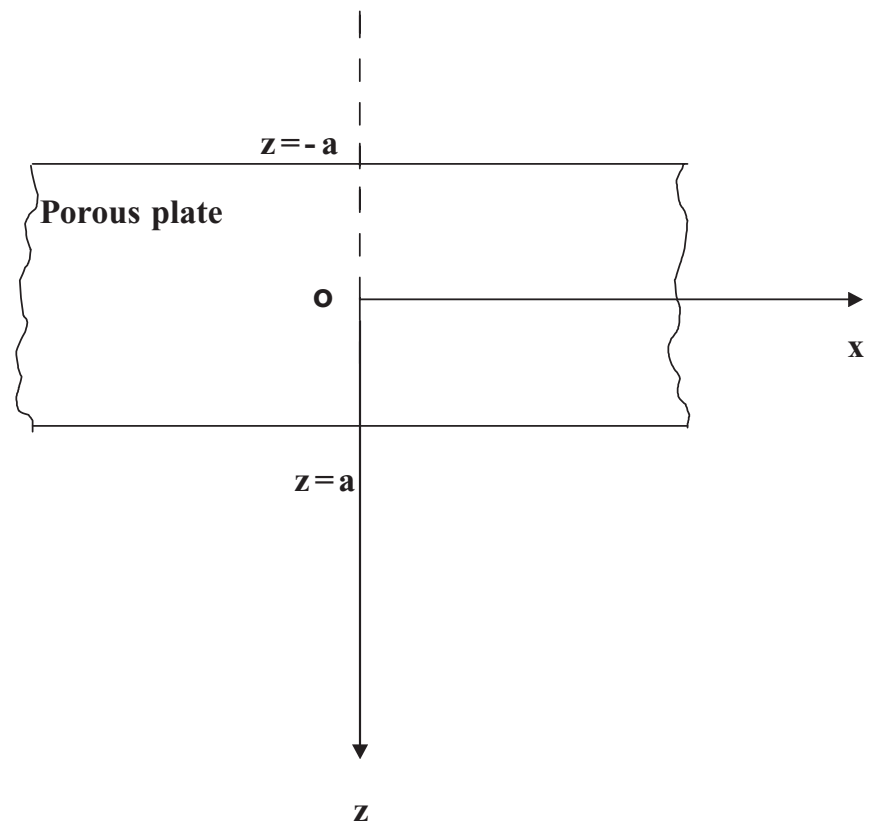

Figure 1. Geometry of the problem.

\& Choudhury (1995) studied the propagation of magneto-elastic shear waves in an infinite self-reinforced plate.

In this paper, we have studied the propagation of shear waves in an infinite fluid-saturated porous transverse-isotropic plate (figure 1). The frequency spectra for $\mathrm{SH}$-modes in the plate are drawn graphically and show that the branches are parabolas. We have also discussed the phase velocities of SH-modes in porous media and observed that the magnitude of the phase velocity decreases as the wave number increases, which means that the thickness of the layer becomes relatively large and the wave propagates in an unbounded medium.

\section{Porous transversely isotropic layer}

The equations of motion for the fluid-saturated layer in the absence of body forces are (Biot 1956, 1962)

$$
\begin{aligned}
\sigma_{i j, j} & =\rho_{11} \ddot{u}_{i}+\rho_{12} \ddot{U}_{i}-b_{i j}\left(\dot{U}_{j}-\dot{u}_{j}\right), \\
\sigma_{, i} & =\rho_{12} \ddot{u}_{i}+\rho_{22} \ddot{U}_{i}-b_{i j}\left(\dot{U}_{j}-\dot{u}_{j}\right),
\end{aligned}
$$

where $\sigma_{i j}$ are the components of stress tensor in solid skeleton. $\sigma=-f p$ is the reduced pressure of the fluid ( $p$ is the pressure in the fluid, and $f$ is the porosity of the medium), $u_{i}$ are the components of the displacement vector of the solid skeleton and $U_{i}$ are those of the fluid. The dynamic coefficients $\rho_{11}, \rho_{22}, \rho_{12}$ taken into account the inertia effects of the moving fluid and are related to the mass densities of the solid $\rho_{s}$ and fluid $\rho_{f}$ by the equations (Biot 1956),

$$
\rho_{11}+\rho_{12}=(1-f) \rho_{s}, \rho_{12}+\rho_{22}=f \rho_{f},
$$


so that mass density of the bulk material is

$$
\rho=\rho_{11}+2 \rho_{12}+\rho_{22}=\rho_{s}+f\left(\rho_{f}-\rho_{s}\right)
$$

The dynamic coefficients, moreover, obey the inequalities (Biot 1956)

$$
\rho_{11}>0, \rho_{22}>0, \rho_{12}<0, \rho_{11} \rho_{22}-\rho_{12}^{2}>0 .
$$

The components of the flow resistance tensor $\mathbf{b}$, for the transverse-isotropy are (Chattopadhyay \& Bandopadhyay 1986; Chattopadhyay \& Choudhury 1995)

$$
\left[b_{i j}\right]=\left[\begin{array}{lll}
b_{11} & 0 & 0 \\
0 & b_{11} & 0 \\
0 & 0 & b_{33}
\end{array}\right]
$$

The stress-strain relations for the transverse-isotropic fluid-saturated porous layer are (Biot 1955, 1962; Achenbach 1976)

$$
\begin{aligned}
& {\left[\begin{array}{l}
\sigma_{11} \\
\sigma_{22} \\
\sigma_{33} \\
\sigma_{23} \\
\sigma_{31} \\
\sigma_{12} \\
\sigma
\end{array}\right]=\left[\begin{array}{lccccccc}
2 N+A & A & F & 0 & 0 & 0 & M & \varepsilon_{11} \\
A & 2 N+A & F & 0 & 0 & 0 & M & \varepsilon_{22} \\
F & F & 2 C & 0 & 0 & 0 & Q & \varepsilon_{33} \\
0 & 0 & 0 & 2 G & 0 & 0 & 0 & \varepsilon_{23} \\
0 & 0 & 0 & 0 & 2 G & 0 & 0 & \varepsilon_{31} \\
0 & 0 & 0 & 0 & 0 & 2 N & 0 & \varepsilon_{12} \\
M & M & Q & 0 & 0 & 0 & R & E
\end{array}\right],} \\
& \text { where } \varepsilon_{i j}=1 / 2\left(u_{i, j}+u_{j, i}\right), E=\operatorname{div} U=U_{i, j}, \varepsilon=\varepsilon_{k, k},
\end{aligned}
$$

and $A, F, C, G, M, Q, N, R$ are the material constants.

On substituting (3) and (4) into (1) we obtain the following system equations

$$
\begin{aligned}
(A \varepsilon+M E)_{, 1}+\left[N\left(u_{1,1}+u_{2,2}\right)+(G+F-A) u_{3,3}\right]_{, 1} & \\
+ & {\left[N\left(u_{1,11}+u_{1,22}\right)+G u_{1,33}\right]=\rho_{11} \ddot{u}_{1}+\rho_{12} \ddot{U}_{1}-b_{11}\left(\dot{U}_{1}-\dot{u}_{1}\right), } \\
(A \varepsilon+ & M E)_{, 2}+\left[N\left(u_{1,1}+u_{2,2}\right)+(G+F-A) u_{3,3}\right]_{, 2} \\
+ & {\left[N\left(u_{2,11}+u_{2,22}\right)+G u_{3,33}\right]=\rho_{11} \ddot{u}_{2}+\rho_{12} \ddot{U}_{2}-b_{11}\left(\dot{U}_{2}-\dot{u}_{2}\right), } \\
G E_{, 3}+ & {\left[(F+G)\left(u_{1,1}+u_{2,2}\right)+C u_{3,3}\right]_{, 3}+\left[G\left(u_{3,11}+u_{3,22}\right)+C u_{3,33}\right] } \\
= & \rho_{11} \ddot{u}_{3}+\rho_{12} \ddot{U}_{3}-b_{33}\left(\dot{U}_{3}-\dot{u}_{3}\right),
\end{aligned}
$$

and

$$
\begin{aligned}
& (M \varepsilon+R E)_{, 1}+(Q-M) u_{3,31}=\rho_{11} \ddot{u}_{1}+\rho_{22} \ddot{U}_{1}+b_{11}\left(\dot{U}_{1}-\dot{u}_{1}\right) \\
& (M \varepsilon+R E)_{, 2}+(Q-M) u_{3,32}=\rho_{11} \ddot{u}_{2}+\rho_{22} \ddot{U}_{2}+b_{11}\left(\dot{U}_{2}-\dot{u}_{2}\right) \\
& (M \varepsilon+R E)_{, 3}+(Q-M) u_{3,33}=\rho_{11} \ddot{u}_{3}+\rho_{22} \ddot{U}_{3}+b_{11}\left(\dot{U}_{3}-\dot{u}_{3}\right) .
\end{aligned}
$$

\section{Formulation and solution of the problem}

We consider the transverse-isotropic fluid-saturated porous plate $-a<z<a$ (figure 1) in the medium. The $x$-axis is chosen parallel to the plate in the direction of propagation of the wave. 
Using the conventional shear-wave conditions

$$
\begin{aligned}
& u_{1} \equiv 0, u_{2}=u_{2}(x, z, t), u_{3} \equiv 0, \\
& U_{1} \equiv 0, U_{2}=U_{2}(x, z, t), U_{3} \equiv 0 .
\end{aligned}
$$

Equations (5) and (6) are reduced to the form

$$
\begin{aligned}
N \frac{\partial^{2} u_{2}}{\partial x^{2}}+G \frac{\partial^{2} u_{2}}{\partial z^{2}} & =\frac{\partial^{2}}{\partial t^{2}}\left(\rho_{11} u_{2}+\rho_{12} U_{2}\right)-b_{11} \frac{\partial}{\partial t}\left(U_{2}-u_{2}\right), \\
0 & =\frac{\partial^{2}}{\partial t^{2}}\left(\rho_{12} u_{2}+\rho_{22} U_{2}\right)+b_{11} \frac{\partial}{\partial t}\left(U_{2}-u_{2}\right) .
\end{aligned}
$$

By eliminating from (8) the functions $u_{2}$ and $U_{2}$ we get

$$
\left[N \frac{\partial^{2} u_{2}}{\partial x^{2}}+G \frac{\partial^{2} u_{2}}{\partial z^{2}}-\left\{\rho_{11} \partial_{t}^{2}+b_{11} \partial_{t}-\frac{\left(\rho_{12} \partial_{t}^{2}-b_{11} \partial_{t}\right)^{2}}{\rho_{22} \partial_{t}^{2}+b_{11} \partial_{t}}\right\}\right]\left(u_{2}, U_{2}\right)=0 \text {, }
$$

where $\partial_{t}=\partial / \partial t$.

For the wave changing harmonically with time, we take

$$
\begin{aligned}
& u_{2}(x, z, t)=u_{2}(z) e^{i(k x-\omega t)}, \\
& U_{2}(x, z, t)=U_{2}(z) e^{i(k x-\omega t)},
\end{aligned}
$$

where $k$ is the wave number and w is the circular frequency.

On substituting (10) into (9), we obtain

$$
\left(\frac{\partial}{\partial z^{2}}+\chi_{1}^{2}\right)\left(u_{2}, U_{2}\right)=0
$$

where $\chi_{1}^{2}$ is a complex quantity and is defined by

$$
\begin{aligned}
\chi_{1}^{2} & =\zeta^{2}-(N / G) k^{2}, \\
\text { and } \zeta^{2} & =\alpha_{1}+i \alpha_{2}, k^{2}=a_{1}+i a_{2}, a_{1} \geq 0, a_{2} \geq 0, \\
\alpha_{1} & =\left(F \omega^{2} / C_{G}^{2}\right), \alpha_{2}=R\left(\omega^{2} / C_{G}^{2}\right), \\
F & =F(\omega)=\frac{1+\Omega^{2} \gamma_{22} C_{1} \gamma_{22}}{1+\left(\Omega \gamma_{22}\right)^{2} C_{1}}, \\
R & =R(\omega)=\frac{\left(\gamma_{22}-C_{1}\right) \Omega}{1+\left(\Omega \gamma_{22}\right)^{2}} \frac{\gamma_{22}}{C_{1}}, \\
C_{1} & =\gamma_{11} \gamma_{22}-\gamma_{12}^{2}, \gamma_{k l}=\rho_{k l} / \rho, k, l=1,2, \\
C_{G}^{2} & =G / \bar{\rho}, \bar{\rho}=\rho_{11}-\left(\rho_{12}^{2} / \rho_{22}\right), \Omega=\rho \omega / b_{11},
\end{aligned}
$$

$\Omega$ is the dimensionless frequency and $C_{G}$ is the velocity of shear waves in the porous plate.

The solutions of (11) are

$$
\begin{aligned}
& u_{2}(x, z, t)=\left(A_{1} \cos \chi_{1} z+A_{2} \sin \chi_{1} z\right) e^{i(k x-\omega t)} \\
& U_{2}(x, z, t)=\left(\bar{A}_{1} \cos \chi_{1} z+\bar{A}_{2} \sin \chi_{1} z\right) e^{i(k x-\omega t)} .
\end{aligned}
$$


The relation between the constants $\bar{A}_{j}$ and $A_{j}(j=1,2)$ provided by ( 8$)$ is

$$
\begin{aligned}
\bar{A}_{j} & =-\left(\beta_{r}-i \beta_{i}\right) A_{j}, \\
\text { where } \quad \beta_{r} & =\frac{\gamma_{11} \gamma_{22} \Omega^{2}-1}{1+\left(\Omega \gamma_{22}\right)^{2}}, \beta_{i}=\frac{\left(\gamma_{12}+\gamma_{22}\right) \Omega}{1+\left(\Omega \gamma_{22}\right)^{2}} .
\end{aligned}
$$

The boundaries $z= \pm a$ are free from traction, i.e.

$$
\sigma_{32}=0, \text { at } z= \pm a \text {. }
$$

Inserting (17) in (20), we get

$$
\begin{aligned}
-A_{1} \sin \chi_{1} a+A_{2} \cos \chi_{1} a & =0, \\
A_{1} \sin \chi_{1} a+A_{2} \cos \chi_{1} a & =0, \\
\text { and }-\bar{A}_{1} \sin \chi_{1} a+\bar{A}_{2} \cos \chi_{1} a & =0, \\
\bar{A}_{1} \sin \chi_{1} a+\bar{A}_{2} \cos \chi_{1} a & =0 .
\end{aligned}
$$

Equations (21) can be satisfied in two ways, either

$$
\begin{aligned}
A_{1} & =0 \text { and } \cos \xi_{1} a=0, \\
\text { or } A_{2} & =0 \text { and } \sin \xi_{1} a=0 .
\end{aligned}
$$

Similarly we solve (22).

For an arbitrary specified value of the wave number $k$, (23) yields an infinite number of solutions for the circular frequency $w$. A specific wave motion of the layer, called mode of propagation, corresponds to each frequency satisfying (17).

If $A_{1}=0$, the expression for $u_{2}(x, z, t)$ shows that the displacement is anti-symmetric with respect to the mid-plane of the layer. The displacement is symmetric if $A_{2}=0$.

In both cases frequencies follow from

$$
\chi_{1} a=m \pi / 2
$$

where $m=0,2,4 \ldots$ (for symmetric modes) and $m=1,3,5 \ldots$ (for anti-symmetric modes).

The real part of (24) is

$$
\Omega_{1}=\left(\frac{m^{2}+(N / G) \xi_{1}}{F-R\left(a_{2} / a_{1}\right)}\right)^{1 / 2}, \frac{C}{C_{G}}=\left(\frac{\left(m^{2} / \xi_{1}\right)+(N / G)}{F-R\left(a_{2} / a_{1}\right)}\right)^{1 / 2}
$$

and imaginary part is

$$
\Omega_{1}=\left(\frac{(N / G) \xi_{1}}{F+R\left(a_{1} / a_{2}\right)}\right)^{1 / 2}, \frac{C}{C_{G}}=\left(\frac{(N / G)}{F+R\left(a_{1} / a_{2}\right)}\right)^{1 / 2}
$$

Taking the modulus from (25) and (26) we have

$$
\begin{aligned}
& \left|\Omega_{1}\right|=\left(\frac{m^{2}+(N / G) \xi_{1}}{F-R\left(a_{2} / a_{1}\right)}+\frac{(N / G) \xi_{1}}{F+R\left(a_{1} / a_{2}\right)}\right)^{1 / 2} \\
& \left|\frac{C}{C_{G}}\right|=\left(\frac{\left(m^{2} / \xi_{1}\right)+(N / G)}{F-R\left(a_{2} / a_{1}\right)}+\frac{(N / G)}{F+R\left(a_{1} / a_{2}\right)}\right)^{1 / 2}
\end{aligned}
$$

where $\Omega_{1}\left(=\left(C^{2} \xi_{1} / C_{G}^{2}\right)^{1 / 2}\right)$, and $C / C_{G}$ and $\xi_{1}\left(=4 a^{2} a_{1} / \pi^{2}\right)$ are dimensionless frequency, phase velocity and wave number respectively.

In the isotropic case $\xi_{1}$ is unity. 


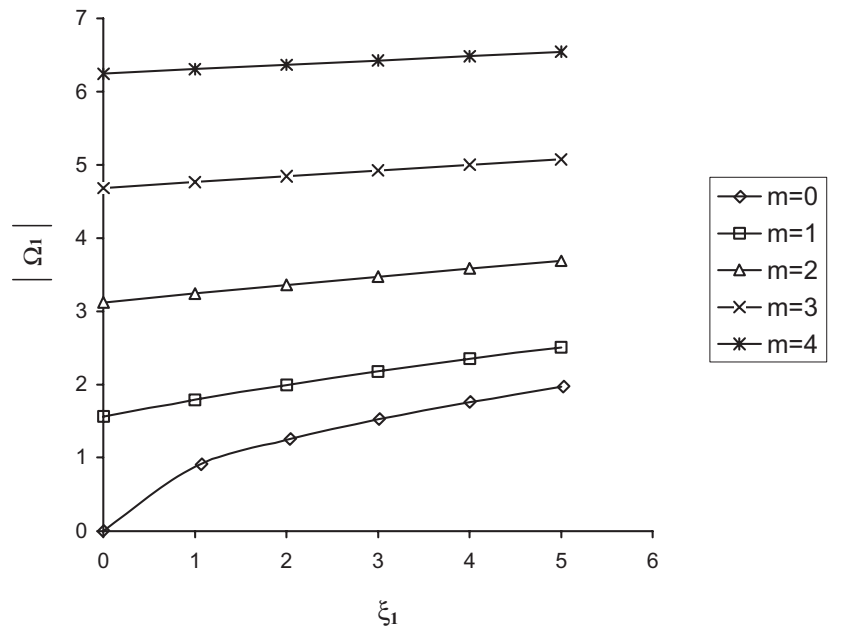

Figure 2. Frequency spectrum for $\mathrm{SH}$-modes when $N / G=1$.

\section{Numerical calculation and discussion}

The frequency and the phase velocity shown in (27) are computed numerically for $a_{2} / a_{1}=0.1$ and $N / G=1,2,3$ at different values of $\xi_{1}$ and for different modes of propagation and are presented in figures $1-8$ and tables 1 and 2 .

The frequency equation in $\left|\Omega_{1}\right|-\xi_{1}$ plane yields an infinite number of continuous curves or branches, each corresponding to an integral value of $m$. A branch displays the relationship between the dimensionless frequency $\left|\Omega_{1}\right|$ and the dimensionless wave number $\xi_{1}$ for a particular mode of propagation. The collection of branches constitutes the frequency spectrum.

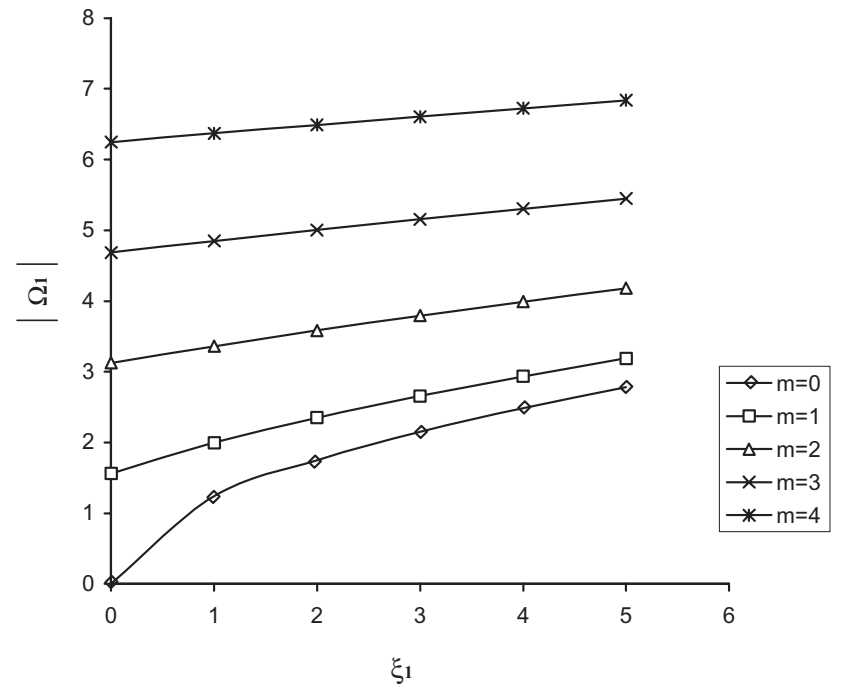

Figure 3. Frequency spectrum for SH-modes when $N / G=2$. 

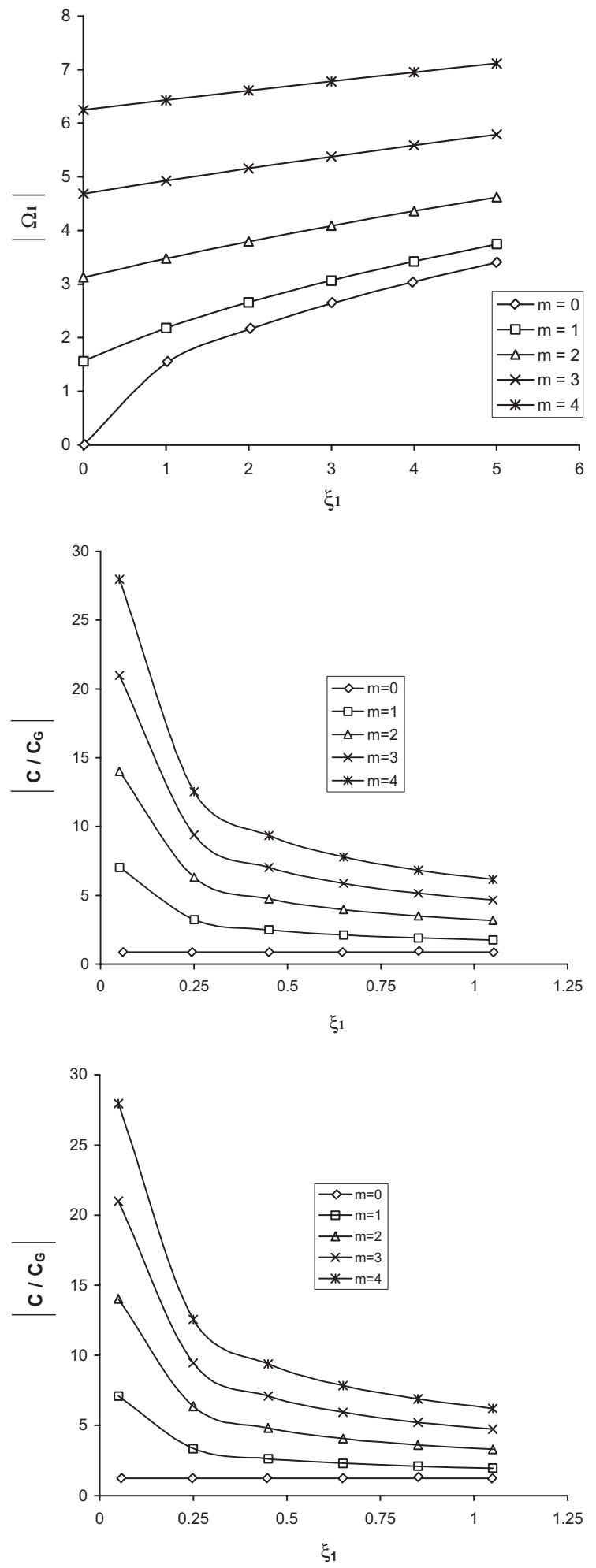

Figure 4. Frequency spectrum for SHmodes when $N / G=3$.

Figure 5. Phase velocity against wave number when $N / G=1$.

Figure 6. Phase velocity against wave number when $N / G=2$. 


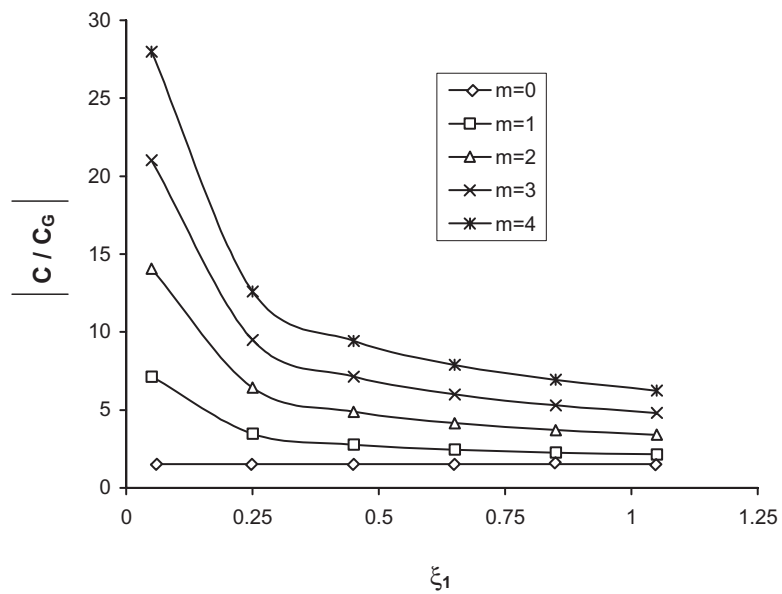

Figure 7. Phase velocity against wave number when $N / G=3$.

For identification of the modes, we call the symmetric SH-modes $(m=0,2,4, \ldots \ldots \ldots)$, the S-modes. Similarly the anti-symmetric SH-modes $(m=1,3,5, \ldots \ldots \ldots)$ are called the AS-modes.

The frequency spectrum for $N / G=1$ is shown in figure 2 . As $\xi_{1}$ increases, the $\left|\Omega_{1}\right|$ also increases. It is noted that when the $N / G=1$, the frequency spectrum for SHmodes behaves as a homogeneous infinite monoclinic crystal plate (Chattopadhyay \& Bandopadhyay 1986). The frequency spectra for $N / G=2,3$ are shown in figure 3 and figure 4.

In figures 2, 3 and 4, when $N / G=1,2,3$ respectively, it is observed that as mode increases the frequency spectrum increases and shows significant increase for the higher modes as compared to the zeroth mode.

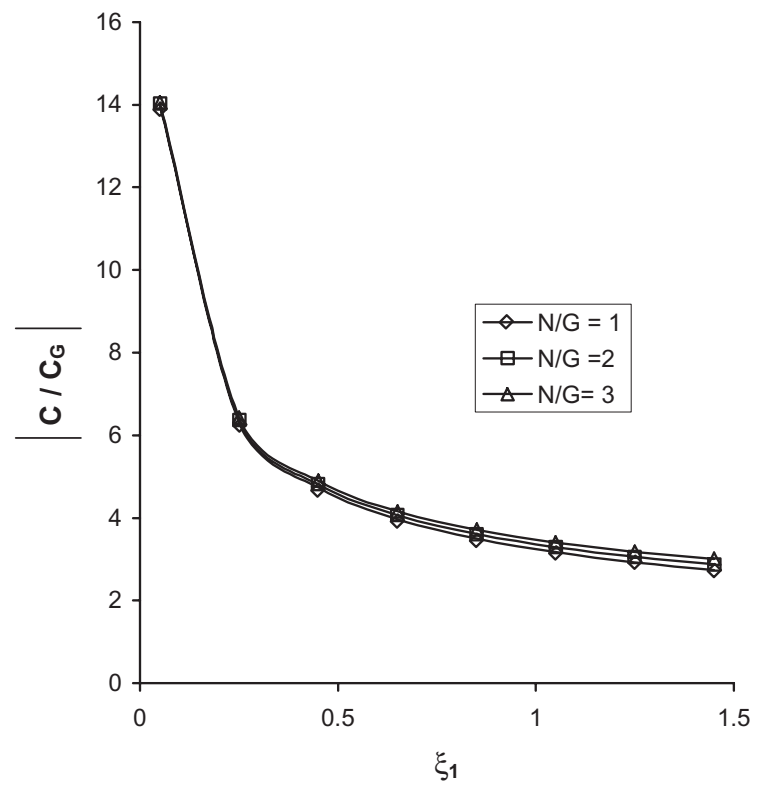

Figure 8. Phase velocity against wave number when $m=2$. 
Table 1. Phase velocity against wave number.

\begin{tabular}{|c|c|c|c|c|c|c|c|}
\hline \multirow{2}{*}{$\frac{N}{G}$} & \multirow{2}{*}{$\begin{array}{l}\left|\frac{C}{C_{G}}\right| \\
\text { for } m=\end{array}$} & \multicolumn{5}{|c|}{$\xi_{1}$} & \multirow[b]{2}{*}{1.05} \\
\hline & & 0.05 & 0.25 & 0.45 & 0.65 & 0.85 & \\
\hline \multirow[t]{5}{*}{1} & 0 & 0.878839 & 0.878839 & 0.878839 & 0.878839 & 0.878839 & 0.878839 \\
\hline & 1 & 7.039378 & 3.244758 & 2.488456 & 2.127135 & 1.90835 & 1.759329 \\
\hline & 2 & 13.99622 & 6.308466 & 4.738415 & 3.972624 & 3.500017 & 3.172362 \\
\hline & 3 & 20.97135 & 9.411548 & 7.039378 & 5.877369 & 5.157259 & 4.655994 \\
\hline & 4 & 27.95103 & 12.52477 & 9.353782 & 7.798069 & 6.832526 & 6.159419 \\
\hline \multirow[t]{5}{*}{2} & 0 & 1.242866 & 1.242866 & 1.242866 & 1.242866 & 1.242866 & 1.242866 \\
\hline & 1 & 7.094026 & 3.361668 & 2.639085 & 2.301534 & 2.100989 & 1.96662 \\
\hline & 2 & 14.02379 & 6.369388 & 4.819225 & 4.068673 & 3.608667 & 3.291844 \\
\hline & 3 & 20.98974 & 9.452498 & 7.094026 & 5.942712 & 5.231604 & 4.738211 \\
\hline & 4 & 27.96484 & 12.55557 & 9.394977 & 7.847435 & 6.888814 & 6.221801 \\
\hline \multirow[t]{5}{*}{3} & 0 & 1.522193 & 1.522193 & 1.522193 & 1.522193 & 1.522193 & 1.522193 \\
\hline & 1 & 7.148256 & 3.474647 & 2.781569 & 2.463619 & 2.277392 & 2.154055 \\
\hline & 2 & 14.0513 & 6.429733 & 4.898703 & 4.162506 & 3.71414 & 3.407139 \\
\hline & 3 & 21.00813 & 9.493258 & 7.148256 & 6.007344 & 5.304907 & 4.819025 \\
\hline & 4 & 27.97865 & 12.58629 & 9.435992 & 7.896493 & 6.944647 & 6.243563 \\
\hline
\end{tabular}

Table 2. Frequency spectrum against wave number.

\begin{tabular}{|c|c|c|c|c|c|c|c|}
\hline \multirow{2}{*}{$\frac{N}{G}$} & \multirow{2}{*}{$\begin{array}{c}\left|\Omega_{1}\right| \text { for } \\
m=\end{array}$} & \multicolumn{5}{|c|}{$\xi_{1}$} & \multirow[b]{2}{*}{5} \\
\hline & & 0 & 1 & 2 & 3 & 4 & \\
\hline \multirow[t]{5}{*}{1} & 0 & 0 & 0.878839 & 1.242866 & 1.522193 & 1.757678 & 1.965143 \\
\hline & 1 & 1.561738 & 1.792033 & 1.995931 & 2.180848 & 2.351267 & 2.510142 \\
\hline & 2 & 3.123475 & 3.244758 & 3.361668 & 3.474647 & 3.584066 & 3.690242 \\
\hline & 3 & 4.685213 & 4.766925 & 4.84726 & 4.926286 & 5.004063 & 5.08065 \\
\hline & 4 & 6.246951 & 6.308466 & 6.369388 & 6.429733 & 6.489516 & 6.548754 \\
\hline \multirow[t]{5}{*}{2} & 0 & 0 & 1.242866 & 1.751678 & 2.152707 & 2.485732 & 2.779132 \\
\hline & 1 & 1.561738 & 1.995931 & 2.351267 & 2.659543 & 2.935624 & 3.187883 \\
\hline & 2 & 3.123475 & 3.361668 & 3.584066 & 3.793447 & 3.991862 & 4.18087 \\
\hline & 3 & 4.685213 & 4.84726 & 5.00406 & 5.1561 & 5.30378 & 5.447458 \\
\hline & 4 & 6.246951 & 6.369388 & 6.489516 & 6.60746 & 6.723336 & 6.837248 \\
\hline \multirow[t]{5}{*}{3} & 0 & 0 & 1.522193 & 2.152707 & 2.636517 & 3.044387 & 3.403728 \\
\hline & 1 & 1.561738 & 2.180848 & 2.659543 & 3.06435 & 3.421596 & 3.744915 \\
\hline & 2 & 3.123475 & 3.474647 & 3.793447 & 4.087459 & 4.361696 & 4.619682 \\
\hline & 3 & 4.685213 & 4.926286 & 5.1561 & 5.376099 & 5.587442 & 5.791078 \\
\hline & 4 & 6.246951 & 6.429733 & 6.60746 & 6.780531 & 6.949294 & 7.114053 \\
\hline
\end{tabular}

Figures 5, 6, 7 show that $\left|\left(C / C_{G}\right)\right|$ decreases as the wave number increases. It is observed that phase velocities are less in porous media than for the elastic isotropic case (Achenbach 1976) for $N / G=1,2,3$ respectively. As $N / G$ increases the phase velocity increases as shown in figure 8. 
It is concluded from the above study that in porous medium the frequency and phase velocity both differs significantly in comparison to that of isotropic elastic medium.

The authors are grateful to Prof S Dey of the Indian School of Mines, Dhanbad for his comments on this paper. The authors also thank Dr S K Mukherjee for his interest and encoragement.

\section{References}

Achenbach J D 1976 Wave propagation in elastic solid (New York: North Holland)

Biot M A 1955 Theory of elasticity and consolidation for a porous an-isotropic solids. J. Appl. Phys. 26: $182-185$

Biot M A 1956 Theory of propagation of elastic waves in a fluid-saturated porous solid. J. Acoust. Soc. Am. 28: 168-178, 179-191

Biot M A 1962 Mechanics of deformation and acoustic propagation in porous medium. J. Appl. Phys. 33: $1482-1498$

Chattopadhyay A, Bandopadhyay U 1986 Shear waves in an infinite monoclinic crystral plate. Int. J. Eng. Sci. 24: 1587-1596

Chattopadhyay A, Choudhury S 1995 Magneto-elastic shear waves in an infinite self-reinforced plate. Int. J. Numer. Anal. Methods Geomech. 19: 289-304

Chattopadhyay A, Chakraborty M, Kushwaha V 1986 On the dispersion equation of Love Waves in a porous layer. Acta Mech. 58: 125-136

Chattopadhyay A, De R K 1983 Love waves in a porous layer with irregular interface. Int. J. Eng. Sci. 21: $1295-1303$

Deresiewicz H 1961 The effect of boundaries on wave propagation in a liquid-filled porous solid: II Love waves in a porous layer. Bull. Seismol. Soc. Am. 51-59

Konczak Z 1989 The propagation of love waves in a fluid-saturated porous anisotropic layer. Acta Mech. 76: 155-168 